\title{
Disconnected diagrams with twisted-mass fermions
}

\section{Abdou Abdel-Rehim ${ }^{a}$, Constantia Alexandrou ${ }^{a b}$, Martha Constantinou $^{a b *}$, Jacob Finkenrath $^{a}$, Kyriakos Hadjiyiannakou ${ }^{c}$, Karl Jansen ${ }^{d}$, Christos Kallidonis ${ }^{a}$, Giannis Koutsou $^{a}$, Alejandro Vaquero Avilés-Casco ${ }^{\dagger} ;$ Julia Volmer $^{d}$}

${ }^{a}$ Computation-based Science and Technology Research Center, CaSToRC

The Cyprus Institute, 20 Kavafi Str. Nicosia 2121, Cyprus

${ }^{b}$ Department of Physics, University of Cyprus

P.O. Box 20537, 1678 Nicosia, Cyprus

${ }^{c}$ The George Washington University

2121 I St NW, Washington, DC 20052, USA

${ }^{d}$ NIC, DESY

Platanenallee 6, D-15738 Zeuthen, Germany

${ }^{e}$ INFN Sezione di Milano-Bicocca

Edificio U2, Piazza della Scienza 3, 20126 Milano, Italy

E-mail: a.abdel-Rehim@cyi.ac.cy, alexandeucy.ac.cy,

marthacetemple.edu, j.finkenrath@cyi.ac.cy,

hadjiyiannakou.kyriakos@ucy.ac.cy, karl.jansen@desy.de,

c.kallidonis@cyi.ac.cy, g.koutsou@cyi.ac.cy,

alexvaq@physics.utah.edu, Julia.Volmer@desy.de

The latest results from the Twisted-Mass collaboration on disconnected diagrams at the physical value of the pion mass are presented. In particular, we focus on the sigma terms, the axial charges and the momentum fraction, all of them for the nucleon. A detailed error analysis for each observable follows, showing the strengths and weaknesses of the one-end trick. Alternatives are discussed.

34th annual International Symposium on Lattice Field Theory

24-30 July 2016

University of Southampton, $U K$

\footnotetext{
${ }^{*}$ Current affiliation: Temple University

${ }^{\dagger}$ Speaker.

${ }^{\ddagger}$ Current affilitaion: Department of Physics and Astronomy, University of Utah
} 


\section{Introduction}

During the last 10 years there has been a tremendous progress in the computation of the disconnected contributions entering the evaluation of nucleon matrix elements. The computation of the all-to-all propagator is the major difficulty in this calculation, but new methods and powerful computers are enabling the computation of fermionic disconnected loops to sufficient accuracy.

In this work we show the final results on the direct evaluation of disconnected loops on a $N_{f}=2$ ensemble at the physical value of the pion mass.

\section{Noise reduction techniques}

The standard approach to compute disconected diagrams involves a stochastic estimation of the all-to-all propagator, generating and inverting random source vectors [1,2]. Usually this method converges poorly, with errors behaving as $O\left(1 / \sqrt{N_{r}}\right)$, where $N_{r}$ is the number of stochastic sources employed, so a large $N_{r}$ is required. But each stochastic source must be inverted through the propagator, making the method extremely expensive in some cases.

\subsection{The Truncated Solver Method (TSM)}

The TSM method [3] allows to circumvent this problem using a predictor-corrector scheme. First we compute a sloppy prediction (low precision LP, as opposed to high precision HP) for the estimate stochastically, and to make it sloppy we invert the source to very low precision. Usually this is achieved by stopping the inverter (a CG in our case) at a fixed value of the residual $\rho_{L P} \gg \rho_{H P}$ or of the number of iterations $n_{L P} \ll n_{H P}$, that is far from convergence. Hence the inversions become cheap and one can increase statistics without incurring in large computer costs.

In order to correct the bias introduced by our sloppy inversions, another set of stochastic sources is generated. Each one of these sources are inverted to both sloppy and high precision, and the difference is calculated. The average over this set of sources gives the correction,

$$
G_{E_{T S M}}:=\underbrace{\frac{1}{N_{\mathrm{HP}}} \sum_{j=1}^{N_{\mathrm{HP}}}\left[\left|s_{j}\right\rangle_{H P}-\left|s_{j}\right\rangle_{L P}\right]\left\langle\eta_{j}\right|}_{\text {Correction }}+\underbrace{\frac{1}{N_{\mathrm{LP}}} \sum_{j=N_{\mathrm{HP}}+1}^{N_{\mathrm{HP}}+N_{\mathrm{LP}}}\left|s_{j}\right\rangle_{L P}\left\langle\eta_{j}\right|}_{\text {Biased estimate }},
$$

Since the correction involves high precision inversions, it is expensive. Nonetheless, if the sloppy and the high precision solutions for the same noise source are closely correlated, only a few rhs are needed to calculate an accurate correction. Then the bulk of the computation goes to the sloppy estimate, and the errors decrease approximately as $\left.O\left(1 / \sqrt{(} N_{L P}\right)\right)$.

As the quark mass decreases, the number of iterations required to achieve a large enough correlation increases. This fact affects negatively the performance of the TSM.

\subsection{Deflation}

Deflation can help overcome the decrease of performance of the TSM for light quark masses by removing the low-modes from the computation: first we calculate the $N_{e v}$ lowest eigenpairs $\lambda_{j},\left|v_{j}\right\rangle$ of $M$. The low-modes generate a subspace where the original noise source can be projected, 
removing the low modes from the stochastic source, and thus leading to a faster inversions due to the improvement conditioning of the matrix. Once inverted, the low-mode part of the solution can be exactly reconstructed from the eigenpairs.

\subsection{Low-mode reconstruction}

The previous method can be further improved if instead of reconstructing $\left|s^{L}\right\rangle$ the all-to-all propagator corresponding to the low-modes is computed [4]. In this method only $\left|s^{D}\right\rangle$ is calculated, contracted and averaged. Then the exact low-mode part of the all-to-all can be reconstructed. This method removes all the stochastic noise coming from the low-modes, but it requires knowledge of the eigenvectors of the full operator, as opposed to the Even-Odd (EO) precondiitoned one. In order to choose the best approach, we tried several combinations summarized in Table 1. In our experience, it was more advantageous to compute the eigenvalues of both operators (full and preconditioned), to reduce the variance of the loops and the cost of the inversions.

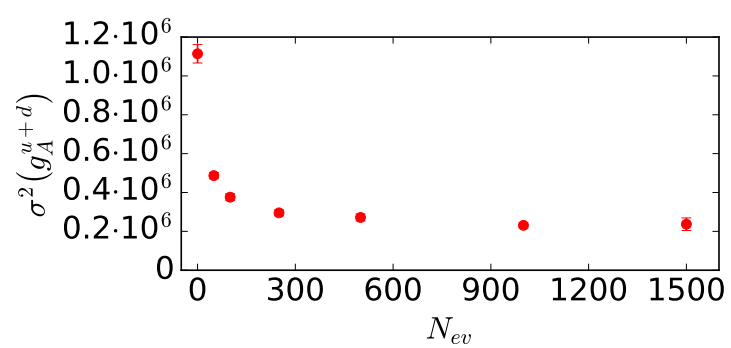

Figure 1: Evolution of the variance for the nucleon $g_{A}^{u+d}$ as the number of low modes for the exact part increases. There is a saturation from $\approx 250$ modes on, where the rest of the modes contribute with similar weights, and there are no further gains. In our tests the most efficient approach didn't reach the saturation point.

\begin{tabular}{l|c|c} 
Method & $N_{s t}$ & Cost \\
\hline$N_{e v}^{E O}=500$ & 2250 & 1.00 \\
$N_{e v}^{F u l l}=100(\mathrm{LM})$ & 750 & 1.54 \\
$N_{e v}^{F u l l}=250(\mathrm{LM})$ & 600 & 0.97 \\
$N_{e v}^{E O}=500, N_{e v}^{\text {Full }}=100(\mathrm{LM})$ & 750 & 0.61 \\
$N_{e v}^{E O}=500, N_{e v}^{F u l l}=250(\mathrm{LM})$ & 600 & 0.77
\end{tabular}

Table 1: Cost and number of rhs required to reach a fixed error in the different deflation schemes, where LM stands for Low Mode reconstruction. The cost is normalized to that of the blue method, which is the one we used for the ultralocal quantities at light masses. The onederivative operators used the more efficient red method.

\subsection{The one-end trick}

The one-end trick $[5,6]$ for disconnected diagrams is a variance reduction technique that can be applied in twisted-mass fermions to reduce the stochastic errors at no extra cost. In the twisted basis, isovector operators can be casted as a product of propagators,

$$
\Gamma\left[G_{u}-G_{d}\right]=-2 i \mu a \Gamma G_{d} \gamma_{5} G_{u}
$$

A similar construction can be found for isoscalar quantities in the twisted-basis. In fig. 2 we assess the performance of the one-end trick for a few observables, with no other variance reduction technique. Of those, only the $\sigma$-term uses the more powerful, isovector version of the trick. As we see, the $\sigma$-term shows a superb performance, and we could cut the cost of the computation by a factor of 10 and still obtain virtually the same errors. The other observables use the less powerful isoscalar version of the trick, which allows us to keep stochastic errors well under control in most cases, being the electromagnetic form factors a notable exception. 

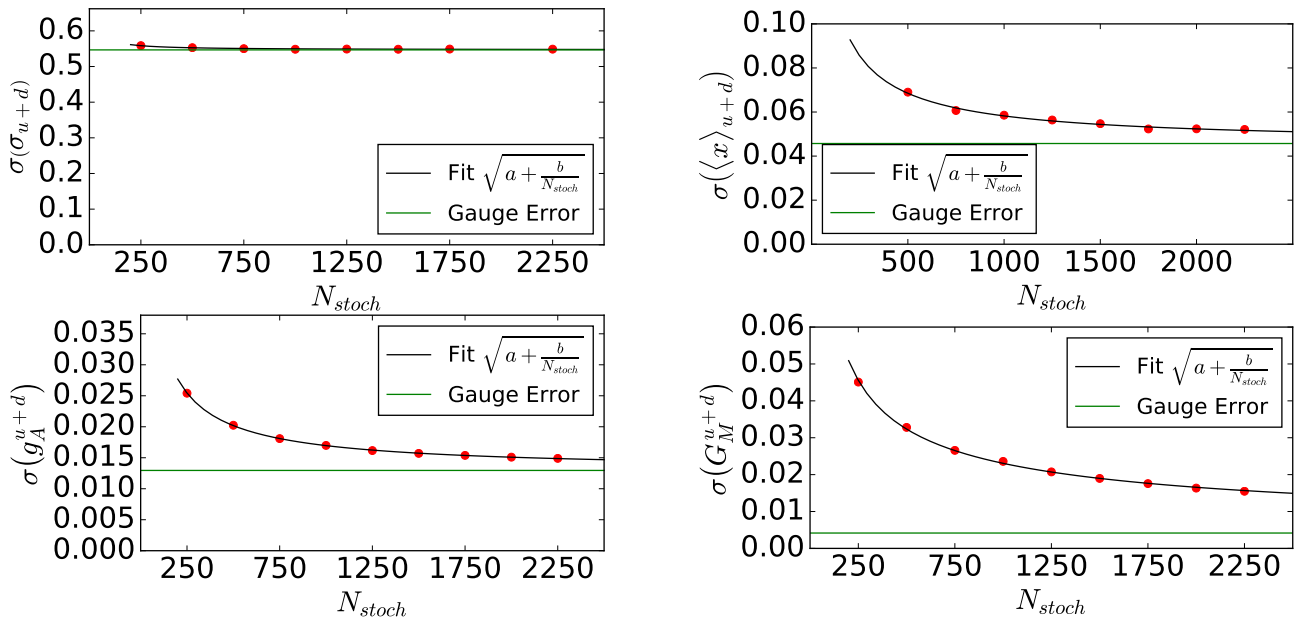

Figure 2: Total absolute error for the disconnected parts of $\sigma_{u+d}, g_{A}^{u+d},\langle x\rangle_{u+d}$, and $G_{M}^{u+d}$, as a function of the number of stochastic sources.

\subsection{Hierarchical probing}

As evidenced by fig.2, the electromagnetic form factors require a different treatment. Probing and hierarchical probing techniques $[7,8]$ have been shown to reduce very effectively the stochastic noise of the electromagnetic form factor.

Since we wanted to keep the one-end trick and to compute the loop in all time-slices for the analysis, we decided to try a 4D hierarchical probing with the one-end trick (in contrast to what is done in [8]) against our current technique (TSM + one-end trick) in an ensemble with a larger pion mass $\left(m_{\pi} \approx 380 \mathrm{MeV}\right)$. We also assessed the effect of spin+color dilution, which is not currently used in our production runs. The results for the nucleon axial charge $g_{A}^{u+d}$ and the magnetic form factor $G_{M}^{u+d}$ are shown in table 2 .

\begin{tabular}{l|c|c} 
Method & $E_{g_{A}}$ & $E_{G_{M}}$ \\
\hline Simple stochastic & $3.073 \pm 0.012$ & $8.85 \pm 0.11$ \\
Hierarchical probing & $2.24 \pm 0.11$ & $114 \pm 2$ \\
Hierarchical probing + dilution & $1.35 \pm 0.05$ & $3.0 \pm 0.4$ \\
TSM & $0.63 \pm 0.07$ & $2.45 \pm 0.04$
\end{tabular}

Table 2: Comparison of $E=\sigma^{2} \times$ Cost for different methods. Lower values are more efficient.

It is notable, in our particular version of this technique, the regression of hierarchical probing without dilution for the magnetic form factor. As a conclusion, we don't see any special advantage with respecto to the TSM, hence for this run we decided to stay with our current methods and ommit the electromagnetic form factors from the calculation.

\section{Details of the simulation and the analysis}

We used a $48^{3} \times 96$ ensemble of $N_{f}=2$ twisted-clover fermions, tuned at the physical value of the light quark mass $\left(m_{\pi} \approx 131 \mathrm{MeV}\right.$ [9]), with $a=0.093 \mathrm{fm}$. Each contribution was com- 
bined with 100 nucleon 2-point functions per configuration. Averaging over forward-backward and proton-neutron resulted in 400 independent measurements per configuration.

The low modes were computed using PARPACK binded to QUDA. Table 1 shows the number of low modes calculated. The stochastic part of the loops as well as the contractions were always computed using QUDA [10, 11].

We used three different analysis methods to remove contamination from the excited states: (1) a plateau fit at fixed sink time, (2) the summation method for several fit ranges and (3) a two-state fit for several values of the sink. In the two-state fit, we would use the same parameters to fit all the plateaux up to the chosen sink. Once the three different estimates were obtained, we tried to find agreement between all the methods, as well as convergence of the final value as we changed the fit parameters and ranges in the three methods.
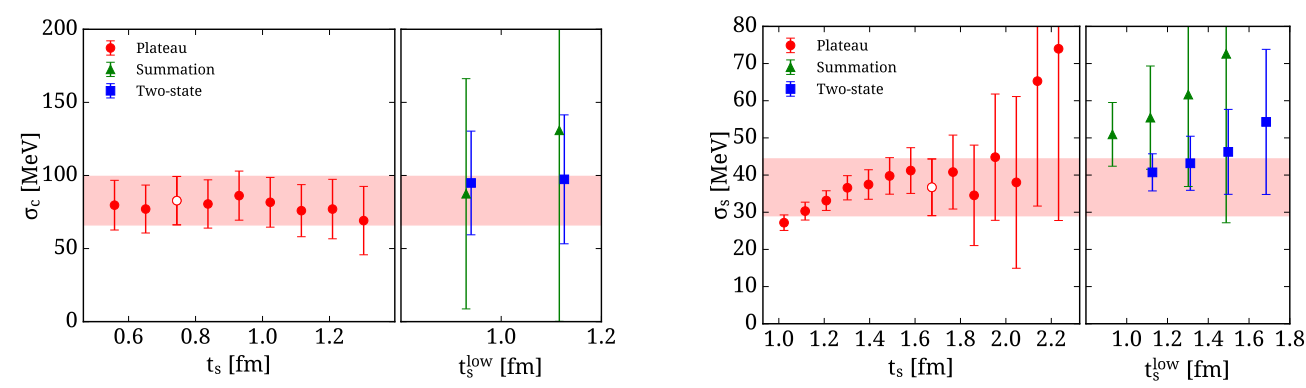

Figure 3: Example of analysis to remove the excited states, with $\sigma_{c}$ on the left and $\sigma_{s}$ on the right. $\sigma_{c}$ is a paradigm of well-behaved quantity, where the three methods agree on a value and converge fast. The case of, $\sigma_{s}$ in contrast, shows a much stronger contribution of excited states, resulting in larger errors in the determination of the final value.

\section{Results}

For each value of the quark mass, we calculated the nucleon $\sigma$-term, axial and tensor charges, and for the light and the strange we also calculated the momentum fraction. In general, we observe large contributions from the excited states coming from the $\sigma$-terms, being $\sigma_{c}$ a notable exception. Other quantities are less prone to these controibutions, but show larger statistical errors.

In general, the $\sigma$-terms and the axial charges give good signal for all the three masses; in particular, the upper plots of fig. 4 and 5 show a clear signal and good convergence. The charm (fig. 6) is noisier, but the absence of contributions of excited states allows us to settle at a much lower value for the sink.

The tensor charge and the momentum fraction are more difficult to compute: for the tensor our signal is compatible with zero within $2 \sigma$ for all the masses, whewreas the momentum fraction gives a clear non-zero signal with large errors.

\section{Discussion}

This work summarizes the effort of the EMTC to calculate disconnected diagrams directly at the physical pion mass, showing results of a high precision computation of the disconnected contribution to all the charges and matrix elements of ultralocal and one-derivative operators for the nucleon, apart from the electromagnetic form factors. Most of our results are in good agreement 

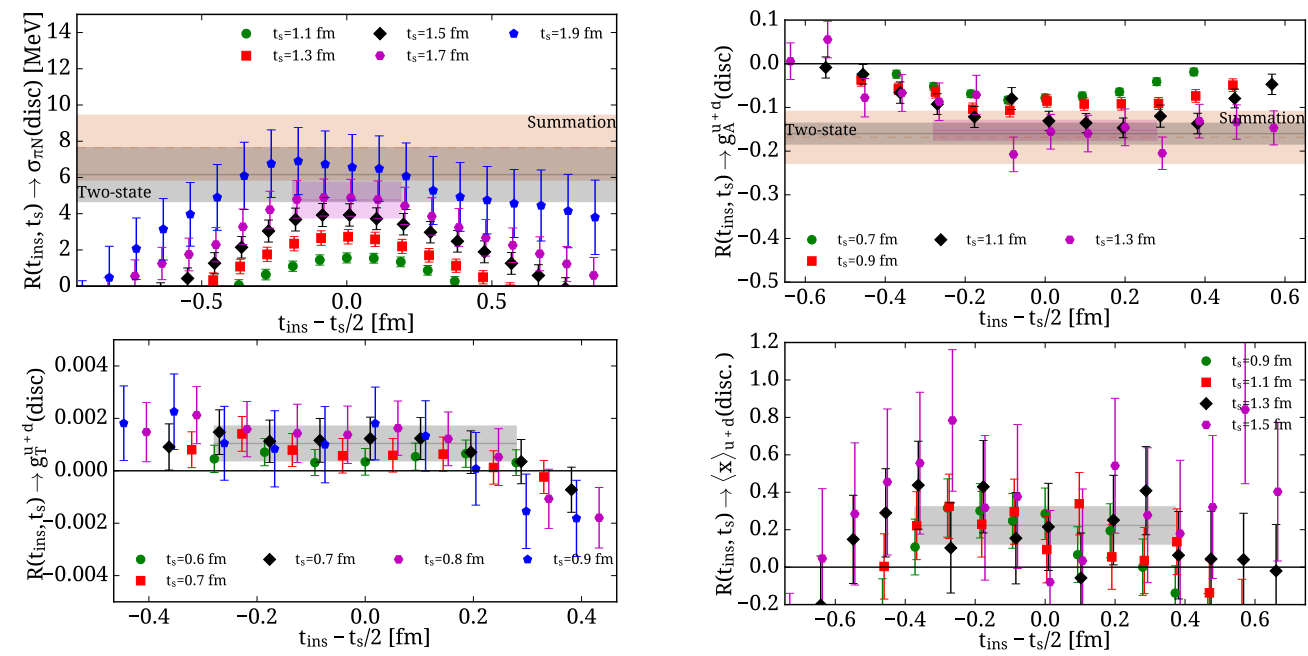

Figure 4: Disconnected quantities for the light quark mass. For the ultralocal we used 2250 stochastic sources accelerated with a deflated solver over 2136 configurations, giving a total of 854400 measurements. The one-derivative results were computed in a new run with low mode reconstruction with 100 eigenvectors, and 1000 stochastic sources using a deflated solver for the high modes, over 1219 configurations, resulting in 487600 measurements.
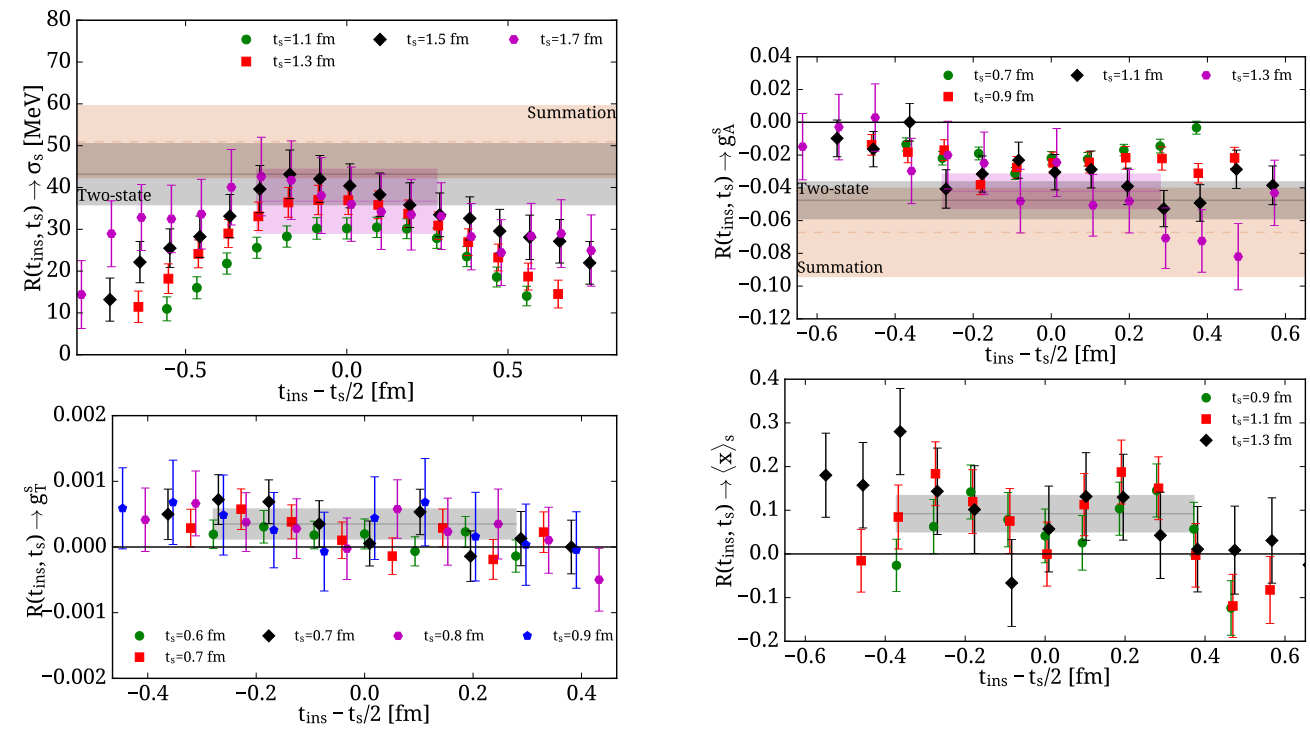

Figure 5: Disconnected quantities for the strange quark mass. Here we drop deflation in favor of the TSM: the ultralocal employed 63HP and 1024LP stochastic sources per configuration, whereas the one-derivative calculation used $30 \mathrm{HP}$ and 960LP sources. The number of configurations for both runs was increased to 2153, resulting in 861200 measurements.

to what other groups have computed, and have led us to draw interesting conclusions on the properties of the nucleon [12]; the rest are new quantities that have never been computed before, thus establishing a new benchmark.

Our next steps are to analyze another two ensembles at the physical pion mass, one $N_{f}=2$ with larger volume, so we can estimate volume effects, and another one with $N_{f}=2+1+1$ in order to assess the errors coming from the removal of the strange and charm sea quarks. We are also currently testing new techniques that should allow us to compute the disconnected electromagnetic form factors with a reasonable stochastic error. 

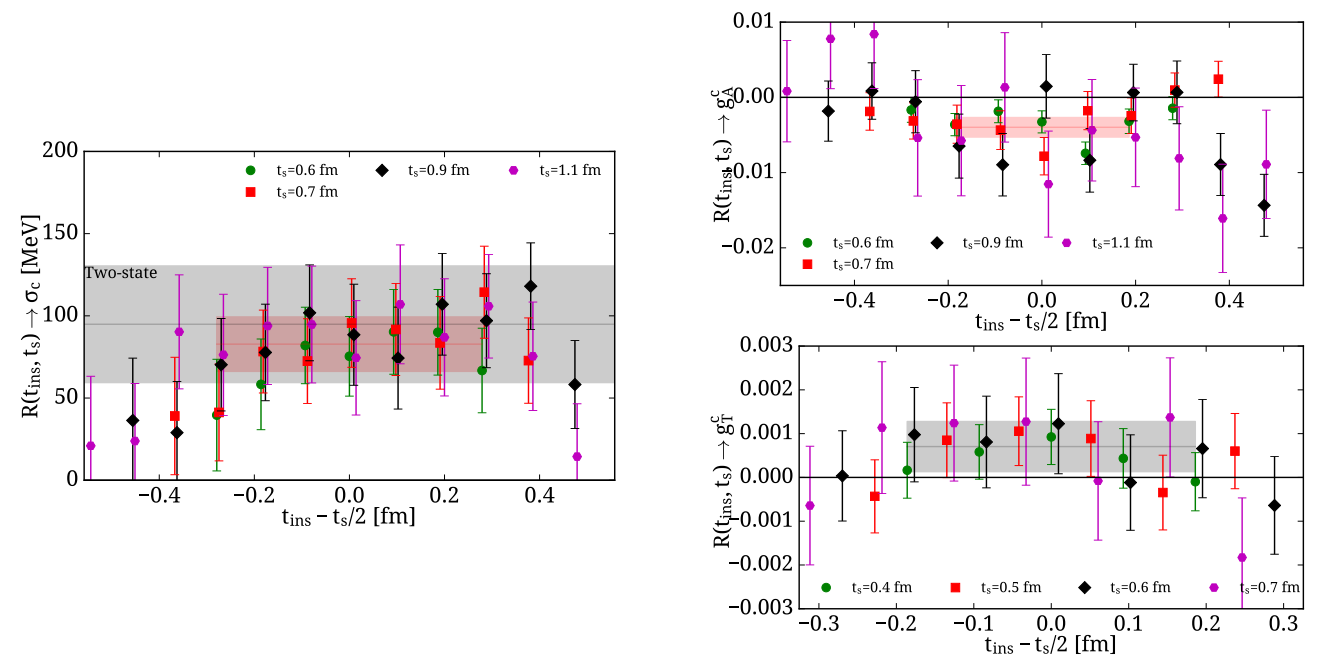

Figure 6: Disconnected quantities for the charm quark mass. As in the strange case, we used solely the TSM as a variance reduction technique, and didn't try to invert faster with deflation. The charm used 5HP and 1250LP per configuration, and the total number of configurations used was 2153 , totaling 861200 measurements.

\section{Acknowledgments}

This work was supported, in part, by a grant from the Swiss National Supercomputing Centre (CSCS) under project ID s625. Additional computational resources were provided by the Cy-Tera machine at The Cyprus Institute funded by the Cyprus Research Promotion Foundation (RPF), NEAYПО $\Delta \mathrm{OMH} / \Sigma T$ TPTH/0308/31.

\section{References}

[1] K. Bitar et al; Nucl. Phys. B313 (1989), 348.

[2] S. Dong, K. Liu; Phys. Lett. B328 (1994), 130, [arXiv:hep-lat/9308015].

[3] G. Bali, S. Collins and A. Schäffer; PoSLaT2007, 141.

[4] H. Neff et al.; Phys. Rev. D64 (2001), 114509, [arXiv:hep-lat/0106016].

G. Bali et al.; Comp. Phys. Comm. 181 (2010), 1570, [arXiv:0910.3970].

[5] M. S. Foster and C. Michael; Phys. Rev. D59 (1999), 074503, [arXiv:hep-lat/9810021].

[6] C. McNeile and C. Michael; Phys. Rev. D73 (2006), 074506, [arXiv:hep-lat/0603007].

[7] E. Endress, C. Pena, K. Sivalingam; Comp. Phys. Comm. 195, (2015), 35, [arXiv:1402.0831].

[8] A. Stathopoulos, J. Laeuchli and K. Orginos; SIAM J. Sci. Comp. 35-5, S299, [arXiv:1302.4018].

J. Green et al.; Phys. Rev. D92 (2015), 031501, [arXiv:1505.01803].

[9] ETM, A. Abdel-Rehim et al., (2015), [arXiv:1507.05068].

[10] M. A. Clark et al., Comput. Phys. Commun. 181 (2010), 1517, [arXiv:0911.3191].

R. Babich et al., SC 2011, arXiv:1109.2935.

[11] C. Alexandrou et al., PoSLaT2013, 411.

[12] C. Alexandrou et al. PoSLaT2016, 153. 